\title{
VECTOR BUNDLES ON COMPLEX PROJECTIVE SPACES AND SYSTEMS OF PARTIAL DIFFERENTIAL EQUATIONS. I
}

\author{
PETER F. STILLER
}

\begin{abstract}
This paper establishes and investigates a relationship between the space of solutions of a system of constant coefficient partial differential equations and the cohomology ( $H^{1}$ in particular) of an associated vector bundle/reflexive sheaf on complex projective space. Using results of Grothendieck and Shatz on vector bundles over projective one-space, the case of partial differential equations in two variables is completely analyzed. The final section applies results about vector bundles on higher-dimensional projective spaces to the case of three or more variables.
\end{abstract}

1. Introduction. In this paper we prove a series of results which establish that an interesting and useful connection exists between local problems in approximation theory, properties of vector bundles on complex projective spaces, and systems of constant coefficient partial differential equations.

As an enticement to the reader (particularly one unfamiliar with the algebrogeometric machinery), we offer a curious result.

THEOREM 1.1. On $\mathbf{R}^{n}, n \geqslant 2$, consider $n$ constant coefficient partial differential operators $p_{i}(\partial)$ having orders $d_{i} \geqslant 1$,

$$
p_{i}(\partial)=\sum_{\substack{\alpha=\left(\alpha_{1} \ldots, \alpha_{n}\right) \\ 0 \leqslant \alpha_{j} \leqslant d_{i} \\ \alpha_{1}+\cdots+\alpha_{n}=d_{i}}} a_{\alpha}^{(i)} \frac{\partial^{d_{i}}}{\partial x_{1}^{\alpha_{1}} \cdots \partial x_{n}^{\alpha_{n}}}, \quad a_{\alpha}^{(i)} \in \mathbf{R}, i=1, \ldots, n .
$$

Let $D=\left\{f\left(x_{1}, \ldots, x_{n}\right) \in \mathscr{C}_{\mathbf{R}^{n}}^{\infty}\right.$ s.t. $\left.p_{i}(\partial) f=0, \ldots, i\right\}$ be the space of $\mathscr{C}^{\infty}$-functions on $\mathbf{R}^{n}$ annihilated by the $n$ operators (the so-called common null space of the $p_{i}(\partial)$ ), then either $\operatorname{dim}_{\mathbf{R}} D=\infty$ or $D$ is finite dimensional with

$$
\operatorname{dim}_{\mathbf{R}} D=\sum_{k=0}^{\infty} \sum_{l=0}^{n} \sum_{1 \leqslant j_{1}<\cdots<j_{l} \leqslant n}(-1)^{l}\left(\begin{array}{c}
k+n-1-\left(d_{j_{1}}+\cdots+d_{j_{l}}\right) \\
n-1
\end{array}\right)
$$

where for $l=0$ we take $\left(\begin{array}{c}k+n-1 \\ n-1\end{array}\right)$, and where we agree that

$$
\left(\begin{array}{c}
k+n-1-\left(d_{j_{1}}+\cdots+d_{j_{l}}\right) \\
n-1
\end{array}\right)=0 \text { when } k-\left(d_{j_{1}}+\cdots+d_{j_{l}}\right)<0 \text {. }
$$

Received by the editors October 23, 1984 and, in revised form, September 23, 1985.

1980 Mathematics Subject Classification (1985 Revision). Primary 14F05, 32L10, 35Exx, 41A15. 
The second case (when $D$ is finite dimensional) is the generic case occurring on an open dense set of choices for the $p_{i}(\partial)$. Essentially, the exceptional cases (when $\operatorname{dim}_{\mathbf{R}} D=$ $\infty)$ occur on a set of measure zero.

Proof. See $§ 5$ below.

For example, on $\mathbf{R}^{4}$ consider four second order partial differential operators with constant coefficients, then $\operatorname{dim}_{\mathbf{R}} D=16$ or $\infty$ and $\operatorname{dim}_{\mathbf{R}} D=16$ is the generic case.

The result, for this example, is equivalent to the fact that the general stable rank two vector bundle $E$ on complex projective three space $\mathbf{P}_{\mathbf{C}}^{3}$ of topological type $c_{1}=0, c_{2}=4, \alpha=1$ has

$$
\operatorname{dim}_{\mathbf{C}} \bigoplus_{m} H^{1}\left(\mathbf{P}_{\mathbf{C}}^{3}, E(m)\right)=16
$$

(see Chang [1]).

This is no accident; one of the main goals of this paper is to establish a relationship between the common null space of a system of constant coefficient partial differential operators and the cohomology ( $H^{1}$ in particular) of certain vector bundles on $\mathbf{P}_{\mathbf{C}}^{n}$. Most of the results are straightforward once the algebrogeometric machinery is available.

These ideas evolved as a result of a number of discussions with Dr. Charles Micchelli, IBM Research, and to him must go the credit for introducing the author to these problems from the point of view of approximation theory. The author has also profited from several discussions with Dr. Klaus Hollig and Dr. Charles Chui of Texas A \& M.

Finally a word of personal thanks must go to my colleague Dr. David L. Johnson for sharing his mathematical interests (especially in complex vector bundles) with me over the years.

2. Differential operators-problems and facts. We wish to consider two easily stated problems-the first dealing with systems of constant coefficient partial differential equations, and the second concerning conformality conditions in approximation theory. Questions of this second type can be reformulated in algebrogeometric terms and are related to certain reflexive sheaves on $\mathbf{P}_{\mathbf{C}}^{n}$, complex projective $n$-space (see Stiller [7] and $\$ 3$ below).

Let $P_{\mathbf{R}}^{k \cdot n}\left(P_{\mathbf{C}}^{k \cdot n}\right)$ denote the space of homogeneous polynomials of degree $k$ in $n$ variables with coefficients in the real numbers $\mathbf{R}$ (complex numbers $\mathbf{C}$ ). Note that

$$
\operatorname{dim}_{\mathbf{R}} P_{\mathbf{R}}^{k, n}=\left(\begin{array}{c}
n+k-1 \\
n-1
\end{array}\right)=\left(\begin{array}{c}
n+k-1 \\
k
\end{array}\right)
$$

and

$$
\operatorname{dim}_{\mathbf{C}} P_{\mathbf{C}}^{k, n}=\left(\begin{array}{c}
n+k-1 \\
n-1
\end{array}\right)=\left(\begin{array}{c}
n+k-1 \\
k
\end{array}\right) .
$$

Let $p_{1}, \ldots, p_{m}$ be homogeneous polynomials of degree $d_{1}, \ldots, d_{m}$ respectively in $\mathbf{R}\left[x_{1}, \ldots, x_{n}\right]$, i.e. $p_{i} \in P_{\mathbf{R}}^{d_{1}, n}$, and let $p_{i}(\partial)$ denote the corresponding linear homogeneous partial differential operator with constant coefficients given by

$$
p_{i}(\partial)=p_{i}\left(\frac{\partial}{\partial x_{1}}, \ldots, \frac{\partial}{\partial x_{n}}\right) \text {. }
$$


We denote by $D$ the common null space of the operators $p_{i}(\partial)$ in the space of real-valued infinitely differentiable functions on $\mathbf{R}^{n}$ :

$$
D=\left\{f \in \mathscr{C}_{\mathbf{R}^{n}}^{\infty}: p_{i}(\partial) f=0 \text { for } i=1, \ldots, m\right\} .
$$

Proposition 2.1 (Micchelli). $\operatorname{dim}_{\mathbf{R}} D<\infty$ if and only if $\left\{z \in \mathbf{C}^{n}-\{(0, \ldots, 0)\}\right.$ s.t. $\left.p_{i}(z)=0, i=1, \ldots, m\right\}=\varnothing$. In other words, $D$ is finite dimensional if and only if the hypersurfaces $p_{i}=0$ in $\mathbf{P}_{\mathbf{C}}^{n-1}$ have empty intersection (requiring in particular at least as many equations as unknowns, i.e. $m \geqslant n$ ).

Proof. If there is a $z_{0} \in \mathbf{C}^{n}-\{(0, \ldots, 0)\}$ with $p_{i}\left(z_{0}\right)=0, i=1, \ldots, m$, then the real (or imaginary) part of any function $\phi\left(z_{0} \cdot x\right), \phi$ holomorphic, is in $D$. (In particular, if $\phi$ is taken to be a polynomial of degree $d$, we see that $D$ includes polynomials of all degrees.)

Conversely, if the $p_{i}$ have no solution in $\mathbf{P}_{\mathbf{C}}^{n}$ then by the Nullstellensatz $x_{i}^{N} \in$ $\left(p_{1}, \ldots, p_{m}\right)$, the ideal generated by the $p_{i}$, for $N$ sufficiently large. It follows that every $f \in D$ is annihilated by every partial derivative of sufficient order (say $\geqslant$ $(N-1) n+1)$ and that $f$ is a polynomial!

PRoposition 2.2 (Micchelli). If $f \in D$ and $f=\sum_{r=0}^{N} f_{r}, f_{r} \in \mathscr{C}_{\mathbf{R}^{n}}^{\infty}$, where each $f_{r}$ is homogeneous of degree $r$ then $f_{r} \in D$.

We define

$$
D_{k}=\left\{p \in P_{\mathbf{R}}^{k \cdot n}: p_{i}(\partial) p=0, i=1, \ldots, m\right\} .
$$

Thus in the case when $D$ is finite dimensional we have $D=\oplus_{k=0}^{\infty} D_{k}$ where $D_{k}=0$ for $k$ sufficiently large, and in the infinite-dimensional case we have

$$
\bigoplus_{k=0}^{\infty} D_{k}=D^{\text {poly }} \subset D
$$

where $D^{\text {poly }}$ is the space of polynomial solutions to our system of partial differential equations.

We now arrive at our first problem:

Problem 1. Find $\operatorname{dim}_{\mathrm{R}} D_{k}$.

To formulate our second problem, we again take $p_{1}, \ldots, p_{m}$ with $p_{i} \in P_{\mathbf{R}^{\prime}}^{d_{i}, n}$ homogeneous polynomials of degree $d_{i}$ in $n$ variables and consider the space $V_{k}$ of polynomial relations of total degree $k$ :

$$
V_{k}=\left\{\left(q_{1}, \ldots, q_{m}\right) q_{i} \in P_{\mathbf{R}}^{k-d_{i} \cdot n}: \sum_{i=1}^{m} q_{i} p_{i}=0\right\} .
$$

Here it is understood that $P_{\mathbf{R}}^{k-d_{l}, n}=0$ if $d_{i}>k$. The spaces $V_{k}$ arise in approximation theory in the context of the conformality conditions and the construction of multivariate splines (see Chui and Wang [2] and Stiller [7]).

Problem 2. Find $\operatorname{dim}_{\mathbf{R}} V_{k}$.

Note that $\operatorname{dim}_{\mathbf{R}} V_{k}$ clearly increases with $k$. We shall see later, in the case $p_{1}, \ldots, p_{m}$ have no common solution in $\mathbf{P}_{\mathbf{C}}^{n-1}$, that $V_{k}$ can be interpreted as $H^{0}\left(\mathbf{P}_{\mathbf{C}}^{n-1}, E(k)\right)$ and $D_{k}$ as $H^{1}\left(\mathbf{P}_{\mathbf{C}}^{n-1}, E(k)\right)$ for a suitable complex vector bundle $E$ on $\mathbf{P}_{\mathbf{C}}^{n-1}$. For now we shall content ourselves with showing that Problem 1 is "equivalent" to Problem 2. 
Consider the linear map

$$
P_{\mathbf{R}}^{k, n} \stackrel{T}{\mapsto} \bigoplus_{i=1}^{m} P_{\mathbf{R}}^{k-d_{1}, n}, \quad p \mapsto\left(\ldots, p_{i}(\partial) p, \ldots\right)
$$

given by our system of differential operators $p_{i}(\partial)$. The null space (kernel) of $T$ is $D_{k}$.

Proposition 2.3 (Micchelli). The null space (kernel) of the adjoint $T^{*}$ can be identified with $V_{k}$. It follows that

$$
\operatorname{dim} V_{k}-\operatorname{dim} D_{k}=\sum_{i=1}^{m}\left(\begin{array}{c}
n+k-d_{i}-1 \\
n-1
\end{array}\right)_{+}-\left(\begin{array}{c}
n+k-1 \\
n-1
\end{array}\right)
$$

(where + means the value is 0 if $k-d_{i}<0$ ).

Proof. We make $P_{\mathbf{R}}^{k, n}$, or rather $P_{\mathbf{R}}^{k, n} \otimes_{\hat{\mathbf{R}}} \mathbf{C} \cong P_{\mathbf{C}}^{k, n}$, into a Hilbert space by defining $\langle p, q\rangle=p(\partial) q$. Note that this is an inner product, because if $p(x)=$ $\sum_{\alpha} p_{\alpha} x^{\alpha}$ and $q(x)=\sum q_{\alpha} x^{\alpha}$ where $\alpha$ is a multi-index $\alpha_{1}, \ldots, \alpha_{n}$ of total degree $|\alpha|=\alpha_{1}+\cdots+\alpha_{n}=k$ then

$$
p(\partial) \bar{q}=\sum_{\alpha, \beta} \bar{q}_{\alpha} p_{\beta} \frac{\partial^{k}}{\partial x^{\beta}} x^{\alpha}=\sum_{\alpha, \beta} \alpha ! \bar{q}_{\alpha} p_{\beta} \delta_{\alpha \beta}=\sum_{\alpha} \alpha ! \bar{q}_{\alpha} p_{\alpha}
$$

where $\alpha !=\alpha_{1} ! \cdots \alpha_{n} !$ (see Stein and Weiss [12, p. 139]).

It follows easily that the adjoint $T^{*}$ of $T$ is given by

$$
T^{*}\left(q_{1}, \ldots, q_{m}\right)=\sum_{i=1}^{m} q_{i} p_{i}
$$

and an index computation gives the relationship between the dimensions.

We remark that the map $T$ decomposes $P_{\mathbf{R}}^{k, n}$ (or $P_{\mathbf{C}}^{k, n}$ ) into an orthogonal direct sum as

$$
P_{\mathbf{R}}^{k, n} \cong \operatorname{ker}(T) \oplus \operatorname{im}\left(T^{*}\right)
$$

which over $\mathbf{C}$ gives

$$
P_{\mathbf{C}}^{k, n} \cong\left(D_{k} \otimes_{\mathbf{R}} \mathbf{C}\right) \oplus I_{k}
$$

where $I=\oplus_{k} I_{k}$ is the homogeneous ideal in $\mathbf{C}\left[x_{1}, \ldots, x_{n}\right]$ generated by $p_{1}, \ldots, p_{m}$.

Propositions 2.1, 2.2, 2.3 and the remark following the proof of Proposition 2.3 above are due to Micchelli and were communicated privately to the author, who wishes to again express his appreciation and gratitude by acknowledging Dr. Micchelli's contribution.

In the case when $D$ is finite dimensional, that is when the $p_{i}$ have no common solution in $\mathbf{P}_{\mathbf{C}}^{n-1}$, we obviously have $D_{k}=0$ for $k$ sufficiently large. Thus

Corollary 2.4 .

$$
\operatorname{dim}_{\mathbf{R}} V_{k}=\sum_{i=1}^{m}\left(\begin{array}{c}
n+k-d_{i}-1 \\
n-1
\end{array}\right)_{+}-\left(\begin{array}{c}
n+k-1 \\
n-1
\end{array}\right)
$$

for $k$ sufficiently large, provided that the $p_{i}$ have no common solution in $\mathbf{P}_{\mathbf{C}}^{n-1}$ (see also Stiller [7, Theorem 3.1]). 
On the other hand in the case $D$ is infinite dimensional, let $X \subset \mathbf{P}_{\mathbf{C}}^{n-1}$ be the nonempty closed subscheme defined by the coherent sheaf of ideals $\mathscr{I}_{X}$ associated to the homogeneous ideal generated by the $p_{i}$ in $\mathbf{C}\left[x_{1} \cdots x_{n}\right]$, then we shall show in the next section that

Corollary 2.5. $\operatorname{dim}_{\mathbf{R}} D_{k}=\operatorname{dim}_{\mathbf{C}} H^{0}\left(X, \mathcal{O}_{X}(k)\right)$ for $k$ sufficiently large. (Of course this is also valid in the case where $D$ is finite dimensional because $X=\varnothing$ and $\operatorname{dim}_{\mathbf{C}} H^{0}\left(X, \mathcal{O}_{X}(k)\right)=0$.)

This corollary also follows from the remark after Proposition 2.3, because for $k$ large $H^{0}\left(\mathbf{P}_{\mathbf{C}}^{n-1}, \mathscr{I}_{X}(k)\right) \cong I_{k}$ and the exact sequence

$$
0 \rightarrow \mathscr{I}_{X}(k) \rightarrow \mathcal{O}_{\mathbf{P}_{\mathrm{C}}^{n-1}}(k) \rightarrow \mathcal{O}_{X}(k) \rightarrow 0
$$

gives an exact sequence of $H^{0}$ 's; recall $H^{0}\left(\mathbf{P}_{\mathbf{C}}^{n-1}, \mathcal{O}_{\mathbf{P}_{\mathrm{C}}^{n-1}}(k)\right)$ can be identified with $P_{\mathbf{C}}^{k, n}$.

As a special case of Corollary 2.5, when $X$ is supported on a finite set of points, we will have $\operatorname{dim}_{\mathbf{R}} D_{k}$ independent of $k$ for $k$ sufficiently large, i.e. constant, because in that case $\mathcal{O}_{X}(k) \cong \mathcal{O}_{X}$. In fact, we can say more in the general case.

Proposition 2.6. $\operatorname{dim}_{\mathbf{R}} D_{k}$ for $k$ large can be given by a polynomial in $k$ with rational coefficients and degree equal to the dimension of $X$.

Proof. We have $\operatorname{dim}_{\mathbf{R}} D_{k}=\operatorname{dim}_{\mathbf{C}} H^{0}\left(X, \mathcal{O}_{X}(k)\right)$ for $k$ large, so the polynomial will be the Hilbert polynomial for $X$ (see Hartshorne [13, Chapter 1, §7]).

In particular for $k$ large $\operatorname{dim}_{\mathbf{R}} D_{k}$ is an increasing function of $k$ so that $\operatorname{dim}_{\mathbf{R}} D_{k} \rightarrow \infty$ as $k \rightarrow \infty$.

In the next section we proceed with the development of this algebrogeometric approach to Problems 1 and 2. A complete answer in the two variable case is discussed in $\$ 4$ below, and some cases in three or more variables are discussed in our last section.

3. Translation to vector bundles/reflexive sheaves. As before, let $p_{1}, \ldots, p_{m}$ be homogeneous polynomials in $\mathbf{R}\left[x_{1} \cdots x_{n}\right], n \geqslant 2$, and say $p_{i}$ has degree $d_{i} \geqslant 1$ so that $p_{i} \in P_{\mathbf{R}}^{d_{i, n}}$. We identify $P_{\mathbf{C}}^{d_{i}, n}$ with $H^{0}\left(\mathbf{P}_{\mathbf{C}}^{n-1}, \mathcal{O}_{\mathbf{P}_{\mathbf{C}}^{n-1}}\left(d_{i}\right)\right)$, the space of global sections of the line bundle $\mathcal{O}_{\mathbf{P}_{\mathbf{C}}^{n-1}}\left(d_{i}\right)$ over complex projective $n-1$ space $\mathbf{P}_{\mathbf{C}}^{n-1}$. Viewed in this way each $p_{i}$ gives rise to an injection

$$
0 \rightarrow \mathcal{O}_{\mathbf{P}_{\mathrm{C}}^{n-1}}\left(-d_{i}\right) \rightarrow \mathcal{O}_{\mathbf{P}_{\mathrm{C}}^{n-1}} .
$$

We consider the direct sum

$$
\bigoplus_{i=1}^{N} \mathcal{O}_{\mathbf{P}_{\mathbf{C}}^{n-1}}\left(-d_{i}\right) \rightarrow \mathcal{O}_{\mathbf{P}_{\mathbf{C}}^{n-1}}
$$

The image of this map is a coherent sheaf of ideals $\mathscr{I}_{X}$ for a closed subscheme $X \subset \mathbf{P}_{\mathbf{C}}^{n-1}$ which is supported on the intersection of the hypersurfaces $p_{i}=0$ in $\mathbf{P}_{\mathbf{C}}^{n-1}$. In fact, $\mathscr{I}_{X}$ is the coherent sheaf associated to the homogeneous ideal generated by the $p_{i}$ in $\mathbf{C}\left[x_{1} \cdots x_{n}\right]$. Note that $\mathscr{I}_{X}=\mathcal{O}_{\mathbf{P}_{\mathbf{C}}^{n-1}}$ when the $p_{i}$ have no common solution in $\mathbf{P}_{\mathbf{C}}^{n-1}$ - that is when $D$, the space of $\mathscr{C}^{\infty}$ solutions $f$ to our 
system of partial differential equations, $p_{i}(\partial) f=0, i=1, \ldots, m$, is finite dimensional.

We consider the sequence

$$
0 \rightarrow K \rightarrow \bigoplus_{i=1}^{m} \mathcal{O}_{\mathbf{P}_{c}^{n} \cdot 1}\left(-d_{i}\right) \rightarrow \mathscr{I}_{X} \rightarrow 0
$$

where $K$ is the kernel (a coherent sheaf on $\mathbf{P}_{\mathbf{C}}^{n-1}$ ) and all the sequences we get after tensoring with the line bundles $\mathcal{O}_{\mathbf{P}_{\mathrm{C}}^{n-1}}(k), k \in \mathbf{Z}$ :

$$
0 \rightarrow K(k) \rightarrow \bigoplus_{i=1}^{m} \mathcal{O}_{\mathbf{P}_{\mathrm{C}}^{n}}\left(k-d_{i}\right) \rightarrow \mathscr{I}_{X}(k) \rightarrow 0 .
$$

Proposition 3.1. $\operatorname{dim}_{\mathbf{C}} H^{0}\left(\mathbf{P}_{\mathbf{C}}^{n-1}, K(k)\right)=\operatorname{dim}_{\mathbf{R}} V_{k}$.

Proof. Taking the long exact cohomology sequence of (2) yields

$$
0 \rightarrow H^{0}(K(k)) \rightarrow H^{0}\left(\bigoplus_{i=1}^{m} \mathcal{O}\left(k-d_{i}\right)\right) \stackrel{\alpha}{\rightarrow} H^{0}\left(\mathscr{I}_{X}(k)\right) \rightarrow \cdots
$$

We identify $H^{0}\left(\oplus_{i=1}^{m} \mathcal{O}\left(k-d_{i}\right)\right)$ with $\oplus_{i=1}^{m} P_{\mathbf{C}}^{k-d_{t}, n}$ and $H^{0}\left(\mathscr{I}_{X}(k)\right) \subset H^{0}(\mathcal{O}(k))$ with the subspace of $P_{\mathbf{C}}^{k . n}$ consisting of those homogeneous polynomials of degree $k$ which "vanish" on $X$ (caution-multiplicities). It is easily seen that $\alpha$ is the map

$$
\bigoplus_{i=1}^{m} P_{\mathbf{C}}^{k-d_{1}, n} \rightarrow P_{\mathbf{C}}^{k, n}, \quad\left(q_{1}, \ldots, q_{m}\right) \mapsto \sum_{i=1}^{m} q_{i} p_{i} .
$$

The result follows.

Proposition 3.2. $K$ is a reflexive sheaf on $\mathbf{P}_{\mathbf{C}}^{n-1}$ and is in fact locally free (vector bundle) in the case $n=2,3$ or in the case $n \geqslant 4$ when the $p_{i}$ have no common solution in $\mathbf{P}_{\mathbf{C}}^{n-1}$.

Proof. To show $K$ is reflexive it is enough to show it it torsion free and normal. Torsion free is obvious, and normality results from the fact that $\mathscr{I}_{X}$ is torsion free (see [5, 1.1.12 Lemma, p. 150 and 1.1.16 Lemma, p. 158]). Now a reflexive sheaf has its singularity set in codimension 3 , so that when $n=2, \mathbf{P}_{\mathbf{C}}^{1}$, or $n=3, \mathbf{P}_{\mathbf{C}}^{2}$, it must be locally free. Finally if the $p_{i}$ have no common solution in $\mathbf{P}_{\mathbf{C}}^{n-1}$ then $K$ is the kernel of a surjective map of locally free sheaves (vector bundles) and hence is locally free.

Proposition 3.3. For $\dot{k}$ sufficiently large

$$
\begin{aligned}
\operatorname{dim}_{\mathbf{C}} H^{0}\left(\mathbf{P}_{\mathbf{C}}^{n-1},\right. & K(k)) \\
= & \sum_{i=1}^{m}\left(\begin{array}{c}
n+k-d_{i}-1 \\
n-1
\end{array}\right)_{+}-\left(\begin{array}{c}
n+k-1 \\
n-1
\end{array}\right)+\operatorname{dim}_{\mathbf{C}} H^{0}\left(\mathcal{O}_{X}(k)\right)
\end{aligned}
$$

where the + indicates that we take

and $\mathcal{O}_{X}$, the cokernel in

$$
\left(\begin{array}{c}
n+k-d_{i}-1 \\
n-1
\end{array}\right)=0 \quad \text { if } k-d_{i}<0
$$

$$
0 \rightarrow \mathscr{I}_{X} \rightarrow \mathcal{O}_{\mathbf{P}_{\mathrm{C}}^{n}} \rightarrow \mathcal{O}_{X} \rightarrow 0
$$

is the structure sheaf of the closed subscheme $X$ in $\mathbf{P}_{\mathbf{C}}^{n-1}$. 
Proof. For $k$ sufficiently large $H^{1}(K(k))=0$ and $H^{1}\left(\mathscr{I}_{X}(k)\right)=0$ and the result follows easily from the usual long exact sequences in cohomology.

Combining this proposition with Proposition 3.1 gives Corollary 2.4 and Corollary 2.5.

We come now to the main result that allows us to translate the two problems of $\S 2$ - namely $\operatorname{dim}_{\mathbf{R}} V_{k}=$ ? and $\operatorname{dim}_{\mathbf{R}} D_{k}=$ ?, into problems about vector bundles/reflexive sheaves on $\mathbf{P}_{\mathbf{C}}^{n-1}$.

THEOREM 3.4. Let $p_{1}, \ldots, p_{m} \in \mathbf{R}\left[x_{1} \cdots x_{n}\right]$ be homogeneous polynomials in $n$ variables of degrees $d_{1}, \ldots, d_{m}$ respectively. Consider the corresponding partial differential operators $p_{i}(\partial) \in \mathbf{R}\left[\partial / \partial x_{1} \cdots \partial / \partial x_{n}\right]$ and let $D$ be the space of $\mathscr{C}_{\mathbf{R}^{\prime \prime}}^{\infty}$ solutions to this system of partial differential equations and let $D_{k}$ be the homogeneous polynomials of degree $k$ in $D$,

$$
D=\left\{f \in \mathscr{C}_{\mathbf{R}^{n}}^{\infty} \text { s.t. } p_{i}(\partial) f=0, i=1, \ldots, m\right\}, \quad D_{k}=D \cap P_{\mathbf{R}}^{k, n} .
$$

If $n \geqslant 3$ and the $p_{i}$ have no common solution in $\mathbf{P}_{\mathbf{C}}^{n-1}$ then we have for all $k$ that

and

$$
\operatorname{dim}_{\mathbf{R}} D_{k}=\operatorname{dim}_{\mathbf{C}} H^{1}\left(\mathbf{P}_{\mathbf{C}}^{n-1}, K(k)\right)
$$

$$
\operatorname{dim}_{\mathbf{R}} D=\sum_{k \geqslant 0} \operatorname{dim}_{\mathbf{R}} D_{k}=\sum_{k \in \mathbf{Z}} \operatorname{dim}_{\mathbf{C}} H^{1}\left(\mathbf{P}_{\mathbf{C}}^{n-1}, K(k)\right)
$$

where $K(k)$ is the sheaf constructed above. (We should remark that

$$
\bigoplus_{k \in \mathbf{Z}} H^{1}\left(\mathbf{P}_{\mathbf{C}}^{n-1}, K(k)\right)
$$

can be viewed as a finite-length graded $\mathbf{C}\left[x_{1} \cdots x_{n}\right]$-module and that this module appears in the vector bundle literature; see for example Hartshorne [4].)

Proof. On $\mathbf{P}_{\mathbf{C}}^{n-1}, n \geqslant 3, H^{1}$ of any line bundle is zero. Thus for all $k \in \mathbf{Z}$ we have

$$
0 \rightarrow H^{0}(K(k)) \rightarrow H^{0}\left(\bigoplus_{i=1}^{m} \mathcal{O}_{\mathbf{P}_{\mathrm{C}}^{n-1}}\left(k-d_{i}\right)\right) \rightarrow H^{0}\left(\mathcal{O}_{\mathbf{P}_{\mathrm{C}}^{n-1}}(k)\right) \rightarrow H^{1}(K(k)) \rightarrow 0
$$

and

$$
\operatorname{dim}_{\mathbf{C}} H^{0}(K(k))-\operatorname{dim}_{\mathbf{C}} H^{1}(K(k))=\sum_{i=1}^{m}\left(\begin{array}{c}
n+k-d_{i}-1 \\
n-1
\end{array}\right)_{+}-\left(\begin{array}{c}
n+k-1 \\
n-1
\end{array}\right) .
$$

We have seen that $\operatorname{dim}_{\mathbf{C}} H^{0}(K(k))=\operatorname{dim}_{\mathbf{R}} V_{k}$ and this coupled with Proposition 2.3 gives

and the result follows.

$$
\operatorname{dim}_{\mathbf{C}} H^{1}(K(k))=\operatorname{dim}_{\mathbf{R}} D_{k}
$$

The case $n=2$ is not covered in the theorem, but in this case the sheaf $K$ is locally free on $\mathbf{P}_{\mathbf{C}}^{1}$ and so by a result of Grothendieck [3] isomorphic to a sum of line bundles $\mathcal{O}_{\mathbf{P}_{\mathbf{C}}^{\mathbf{1}}}\left(a_{i}\right)$. Hence $\operatorname{dim}_{\mathbf{R}} V_{k}$ can be determined directly and from that $\operatorname{dim}_{\mathbf{R}} D_{k}$ and $\operatorname{dim}_{\mathbf{R}} D$ (see the next section).

4. Systems of constant coefficient partial differential equations in two variables. Let $p_{1}, \ldots, p_{m} \in \mathbf{R}[x, y]$ be homogeneous polynomials of degree $d_{1}, \ldots, d_{m}$ respectively. For convenience, we order the $p_{i}$ so that $1 \leqslant d_{1} \leqslant d_{2} \leqslant \cdots \leqslant d_{m}$. We adopt the convention that $D_{k}, V_{k}$ and $P_{\mathbf{R}}^{k .2}$ are zero when $k<0$. 
Recall our two questions: Find $\operatorname{dim}_{\mathbf{R}} D_{k}$ and find $\operatorname{dim}_{\mathbf{R}} V_{k}$. Using a result of Grothendieck [3] on the structure of vector bundles over $\mathbf{P}_{\mathbf{C}}^{1}$, and the results of Shatz [6] on subbundles of vector bundles over $\mathbf{P}_{\mathbf{C}}^{1}$, we can say a great deal about these questions in the two variable case.

First, we have shown

$$
\operatorname{dim}_{\mathbf{R}} V_{k}-\operatorname{dim}_{\mathbf{R}} D_{k}=\sum_{i=1}^{m}\left(k-d_{i}+1\right)_{+}-(k+1)
$$

where the + means the value is 0 if $k-d_{i}<0$. Second, if the $p_{i}$ have a greatest common factor $p \in P_{\mathbf{R}}^{d .2}$ of degree $d \geqslant 1$ then

$$
\begin{gathered}
\operatorname{dim}_{\mathbf{R}} D=\infty, \\
\operatorname{dim}_{\mathbf{R}} D_{k}=d \text { for } k \text { sufficiently large, }
\end{gathered}
$$

(because the scheme $X \subset \mathbf{P}_{\mathbf{C}}^{1}$ consists of $d$ points if we count with multiplicity). We can determine $\operatorname{dim}_{\mathbf{R}} V_{k}$ by considering the analogous problem for $\tilde{p}_{i}=p_{i} / p \in$ $P_{\mathbf{R}^{\prime}}^{d_{1}-d .2}$. We will have $V_{k}=\tilde{V}_{k-d}$ where

$$
\tilde{V}_{k-d}=\left\{\left(q_{1}, \ldots, q_{m}\right) q_{i} \in P_{\mathbf{R}}^{k-d_{i}}: \sum_{i=1}^{m} q_{i} \tilde{p}_{i}=0\right\} .
$$

In fact, we will also have in this case that

$$
\begin{aligned}
\operatorname{dim}_{\mathbf{R}} D_{k} & =\operatorname{dim}_{\mathbf{R}} \tilde{D}_{k-d}+d \text { for } k \geqslant d, \\
& =k+1 \text { for } 0 \leqslant k<d .
\end{aligned}
$$

Thus we can always reduce the case where the $p_{i}$ have a common factor to one where they do not, and so from now on in our discussion of the two variable case we shall assume $p_{1}, \ldots, p_{m}$ have no common factor. In this case we have, for every $k$, the exact sequence of vector bundles (see $\S 3$ above):

$$
0 \rightarrow K(k) \rightarrow \bigoplus_{i=1}^{m} \mathcal{O}_{\mathbf{P}_{\mathrm{C}}^{\prime}}\left(k-d_{i}\right) \rightarrow \mathcal{O}_{\mathbf{P}_{\mathrm{C}}^{\prime}} l(k) \rightarrow 0,
$$

where $H^{0}\left(\mathbf{P}_{\mathbf{C}}^{1}, K(k)\right)$ can be identified with $V_{k} \otimes_{\mathbf{R}} \mathbf{C}$. In particular $K$ is the kernel of the map from $\oplus_{i=1}^{m} \mathcal{O}\left(-d_{i}\right)$ onto $\mathcal{O}$ determined by the $p_{i}$,

$$
0 \rightarrow K \rightarrow \bigoplus_{i=1}^{m} \mathcal{O}\left(-d_{i}\right) \rightarrow \mathcal{O} \rightarrow 0 .
$$

By a theorem of Grothendieck [3] the vector bundle $K$ is isomorphic to a direct sum of line bundles (unique up to the order of the factors)

$$
K=\bigoplus_{j=1}^{m-1} \mathcal{O}\left(-a_{j}\right) .
$$

Elementary considerations show that $\sum_{j=1}^{m-1} a_{j}=\sum_{i=1}^{m} d_{i}$ and $a_{j} \geqslant d_{1} \geqslant 1$. If we could determine the $m-1$ numbers $a_{j}$ we would then have

$$
\operatorname{dim}_{\mathbf{R}} V_{k}=\sum_{j=1}^{m-1}\left(k-a_{j}+1\right)_{+}
$$

and

$$
\operatorname{dim}_{\mathbf{R}} D_{k}=\sum_{j=1}^{m-1}\left(k-a_{j}+1\right)_{+}-\sum_{i=1}^{m}\left(k-d_{i}+1\right)_{+}+k+1 .
$$


Note that for $k+1 \geqslant \max \left\{a_{j}\right.$ 's, $d_{i}$ 's $\}$, we have

$$
\operatorname{dim}_{\mathbf{R}} D_{k}=\sum_{i=1}^{m} d_{i}-\sum_{j=1}^{m} a_{j}=0 \text {. }
$$

In order to give a satisfactory answer, we must determine which sequences of positive integers $1 \leqslant a_{1} \leqslant \cdots \leqslant a_{m-1}$ occur. This is answered by the following:

DEFINITION 4.1. A sequence $1 \leqslant a_{1} \leqslant \cdots \leqslant a_{m-1}$ of positive integers is said to be admissible for a given sequence $1 \leqslant d_{1} \leqslant \cdots \leqslant d_{m}$ if

(1) $\sum_{j=1}^{m-1} a_{j}=\sum_{i=1}^{m} d_{i}$,

(2) $a_{j} \geqslant d_{j}, j=1, \ldots, m-1$,

(3) if $l$ is the least index for which $a_{l} \neq d_{l}$ then for $j \geqslant l$ we should have $a_{j} \geqslant d_{j+1}$.

Finally, the generic admissible sequence for a given sequence $1 \leqslant d_{1} \leqslant \cdots \leqslant d_{m}$ is that admissible sequence which is largest in the lexicographical ordering-i.e. $\left(a_{1} \cdots a_{m-1}\right) \leqslant\left(a_{1}^{\prime} \cdots a_{m-1}^{\prime}\right)$ if the first nonzero difference $a_{j}^{\prime}-a_{j}$ is positive.

THEOREM 4.2. Given $p_{1}, \ldots, p_{m}$ homogeneous polynomials in $\mathbf{R}[x, y]$ with no common factor as above, we have

$$
\operatorname{dim}_{\mathbf{R}} V_{k}=\sum_{j=1}^{m-1}\left(k-a_{j}+1\right)_{+}
$$

and

$$
\operatorname{dim}_{\mathbf{R}} D_{k}=\sum_{j=1}^{m-1}\left(k-a_{j}+1\right)_{+}-\sum_{i=1}^{m}\left(k-d_{i}+1\right)_{+}+k+1
$$

for some admissible sequence $1 \leqslant a_{1} \leqslant \cdots \leqslant a_{m-1}$. Conversely, every admissible sequence arises in this way for some choice of $p_{1}, \ldots, p_{m}$ (possible some $\left.p_{i} \equiv 0\right)$. Moreover, in the space $\bigoplus_{i=1}^{m} P_{\mathbf{R}_{i}, 2}^{d_{1}} \cong \mathbf{R}^{\sum_{i-1}^{m}\left(d_{i}+1\right)}$ of possible choices for the $p_{i}$ there is an open dense set (complement has measure zero and is cut out by polynomial equations) of choices having no common factor for which the generic admissible sequence occurs.

Proof. We need to determine all possible subbundles of $\oplus_{i=1}^{m} \mathcal{O}\left(-d_{i}\right)$ of rank $m-1$ having quotient isomorphic to $\mathcal{O}$. This is done by Shatz in [6]. The statement about the generic case is straightforward.

The generic admissible sequence gives the smallest possible dimension for the space $D$ given the orders (or degrees) $d_{i}$ of the partial differential operators $p_{i}(\partial)$ (or polynomials $p_{i}$ ).

We conclude this section with a number of examples. As usual $p_{i}, i=1, \ldots, m$, will be a homogeneous polynomial of degree $d_{i}$ in $\mathbf{R}[x, y]$. We will assume, with no loss of generality, that the $p_{i}$ are ordered so that $1 \leqslant d_{i} \leqslant \cdots \leqslant d_{m}$, and that the $p_{i}$ have no common factor.

Cases. $m=2$. In this case $K \cong \mathcal{O}\left(-d_{1}-d_{2}\right)$ and $\operatorname{dim} V, \operatorname{dim} D_{k}$, and $\operatorname{dim} D$ are easily computed. 
$m=3$. We divide the case of three polynomials into three subcases:

Subcase (1). $d_{3} \geqslant d_{1}+d_{2}$.

The generic sequence is $a_{1}=d_{1}+d_{2}, a_{2}=d_{3}$, and we can show that this occurs if and only if $p_{1}, p_{2}$ have no common factor.

This in the generic case, we have $K \cong \mathcal{O}\left(-d_{1}-d_{2}\right) \oplus \mathcal{O}\left(-d_{3}\right)$.

Subcase (2). $d_{3}=d_{1}+d_{2}-1$.

This is similar to Subcase (1) above except that the last listed sequence $a_{1}=d_{1}+$ $d_{2}, a_{2}=d_{3}$ does not occur and the generic sequence will instead be $a_{1}=d_{1}+d_{2}-1$ $\left(=d_{3}\right) a_{2}=d_{3}+1\left(=d_{1}+d_{2}\right)$. Note however that we still have

$$
K \cong \mathcal{O}\left(-d_{1}-d_{2}\right)+\mathcal{O}\left(-d_{3}\right)
$$

in the generic case and so the formulas for $\operatorname{dim} V_{k}, \operatorname{dim} D_{k}$, and $\operatorname{dim} D$ would remain as in Subcase (1). One can show that the generic case occurs if and only if $p_{1}, p_{2}$ have no common factor or $p_{1}, p_{2}$ have a common factor of degree 1 (which necessarily does not divide $p_{3}$ ).

Subcase (3). $d_{1}+d_{2}-2 \geqslant d_{3}$ (note $d_{1} \geqslant 2$ necessarily).

The generic admissible sequences are

$$
\begin{aligned}
& a_{1}=\left[\frac{d_{1}+d_{2}+d_{3}}{2}\right], \\
& a_{2}=\left[\frac{d_{1}+d_{2}+d_{3}}{2}\right]+\begin{array}{ll}
1 & \text { if } d_{1}+d_{2}+d_{3} \text { is odd, } \\
0 & \text { if } d_{1}+d_{2}+d_{3} \text { is even, }
\end{array}
\end{aligned}
$$

where $\left[\left(d_{1}+d_{2}+d_{3}\right) / 2\right]$ is the largest integer less than or equal to $\left(d_{1}+d_{2}+d_{3}\right) / 2$. It is more difficult in this instance to characterize when the generic case occurs. Specifically, for the generic case we obtain

$$
\begin{aligned}
& \operatorname{dim} V_{k}= \begin{cases}0, & k<\left[\frac{d_{1}+d_{2}+d_{3}}{2}\right], \\
2 k-\sum_{i=1}^{3} d_{i}+2, & k \geqslant\left[\frac{d_{1}+d_{2}+d_{3}}{2}\right],\end{cases} \\
& \operatorname{dim} D_{k}= \begin{cases}k+1-\sum_{i=1}^{3}\left[k-d_{i}+1\right]_{+}, & k<\left[\frac{d_{1}+d_{2}+d_{3}}{2}\right], \\
0, & k \geqslant\left[\frac{d_{1}+d_{2}+d_{3}}{2}\right],\end{cases} \\
& \operatorname{dim} D= \begin{cases}\frac{2 d_{1} d_{2}+2 d_{2} d_{3}+2 d_{1} d_{3}-\sum_{i=1}^{3} d_{i}^{2}}{4} \quad \text { when } d_{1}+d_{2}+d_{3} \text { is even, } \\
\frac{2 d_{1} d_{2}+2 d_{2} d_{3}+2 d_{1} d_{3}-\sum_{i=1}^{3} d_{i}^{2}+1}{4} & \text { when } d_{1}+d_{2}+d_{2} \text { is odd }\end{cases}
\end{aligned}
$$

Next we consider a special case of a more general example arising from the theory of box splines (see Micchelli and Dahman [9, 10, and 11]) where the operators are certain compositions of directional derivatives in $\mathbf{R}^{n}$ (with possible "multiplicities"). Let $p_{1}=x^{m_{1}} y^{m_{2}}, p_{2}=x^{m_{1}}(x+y)^{m_{3}}, p_{3}=y^{m_{2}}(x+y)^{m_{3}}, m_{i} \geqslant 1$, where $m_{1} \leqslant m_{2}$ $\leqslant m_{3}$. We are in Subcase (3) above as

$$
d_{3}=m_{2}+m_{3} \leqslant d_{1}+d_{2}-2=2 m_{1}+m_{2}+m_{3}-2 \text {. }
$$


An easy divisibility argument shows that there can be no relation in total degree $<\left[\left(d_{1}+d_{2}+d_{3}\right) / 2\right]=m_{1}+m_{2}+m_{3}$. We must therefore be in the generic case, and using our formula $\operatorname{dim} D=m_{1} m_{2}+m_{2} m_{3}+m_{1} m_{3}$ which is equal to the number of ways of picking a basis for $\mathbf{R}^{2}$ from the vectors

$$
\underbrace{\left[\begin{array}{l}
1 \\
0
\end{array}\right], \ldots,\left[\begin{array}{l}
1 \\
0
\end{array}\right]}_{m_{1} \text { times }}, \underbrace{\left[\begin{array}{l}
0 \\
1
\end{array}\right], \ldots,\left[\begin{array}{l}
0 \\
1
\end{array}\right]}_{m_{2} \text { times }}, \underbrace{\left[\begin{array}{l}
1 \\
1
\end{array}\right], \ldots,\left[\begin{array}{l}
1 \\
1
\end{array}\right]}_{m_{3} \text { times }} .
$$

Micchelli and Dahman [11] have a general result for more vectors in more variables, and in the sequel to this paper we plan to examine the resulting vector bundles on $\mathbf{P}_{\mathbf{C}}^{n-1}$.

Finally we consider the case $p_{i}=\left(\alpha_{i} x+\beta_{i} y\right)^{d}, i=1, \ldots, m, m \geqslant 2$, where the vectors $\left(\alpha_{i}, \beta_{i}\right)$ in $\mathbf{R}^{2}$ are pairwise linearly independent. Results in Stiller [7] and Chui and Wang [2] show that this case is generic.

5. Case of $>2$ variables. The case of more than two variables is one that is extremely interesting because of its relationship to vector bundles and reflexive sheaves on $\mathbf{P}_{\mathbf{C}}^{n-1}, n \geqslant 3$. There has been a great deal of work done on vector bundles on projective spaces in the last 10 years or so-some of it motivated by connections with mathematical physics (instantons). In particular, there is now a wealth of results available in the cases of $\mathbf{P}_{\mathbf{C}}^{2}$ and $\mathbf{P}_{\mathbf{C}}^{3}$. Less is known in higher dimensions. In this paper we have dealt mostly with using vector bundle techniques to answer questions of relevance to partial differential equations and in approximation theory. In our sequel to this paper, we plan to turn the process around and use results in p.d.e. and approximation theory to say something about vector bundles.

We close with the proof of Theorem 1.1 in the Introduction. This result illustrates how to make use of the known facts about vector bundles to obtain results in $n \geqslant 3$ variables. Other examples can be manufactured using other classes of vector bundles.

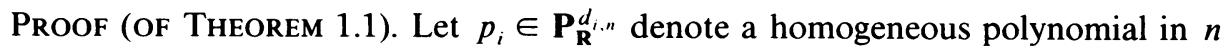
variables of degree $d_{i}$, for $i=1, \ldots, n$, and let $K$ denote the locally free sheaf

$$
0 \rightarrow K \rightarrow \bigoplus_{i=1}^{n} \mathcal{O}_{\mathbf{P}_{\mathrm{C}}^{n-1}}\left(-d_{i}\right)^{\phi} \rightarrow \mathcal{O}_{\mathbf{P}_{\mathrm{C}}^{n-1}} \rightarrow 0
$$

which is the kernel of the map $\phi$ induced by the $p_{i}$. (We are assuming that we are in the case $\operatorname{dim}_{\mathbf{R}} D<\infty$.)

By Proposition 2.3 and Proposition 3.2, it is enough to show that

$$
\begin{aligned}
\operatorname{dim}_{\mathbf{R}} V_{k} & =\operatorname{dim}_{\mathbf{C}} H^{0}\left(\mathbf{P}_{\mathbf{C}}^{n-1}, K(k)\right) \\
& =\sum_{l=2}^{n} \sum_{1 \leqslant j_{1}<\cdots<j_{l} \leqslant n}(-1)^{l}\left(\begin{array}{c}
k+n-1-\left(d_{j_{1}}+\cdots+d_{j_{l}}\right) \\
n-1
\end{array}\right) .
\end{aligned}
$$

In order to show this, let $F$ denote the sheaf $\oplus_{i=1}^{n} \mathcal{O}_{\mathbf{P}_{C}^{n-1}}\left(k-d_{i}\right)$. We then have the Koszul resolution (see Hartshorne [13])

$$
0 \rightarrow \wedge^{n} F \rightarrow \wedge^{n-1} F \rightarrow \cdots \rightarrow \wedge^{2} F \rightarrow \wedge^{1} F=F \stackrel{\phi}{\rightarrow} \mathcal{O}_{\mathbf{P}_{\mathbf{C}}^{n-1}} \rightarrow 0 .
$$


It follows that $K(k)$ has a resolution

$$
0 \rightarrow\left(\wedge^{n} F\right)(k) \rightarrow\left(\wedge^{n-1} F\right)(k) \rightarrow \cdots \rightarrow\left(\wedge^{2} F\right)(k) \rightarrow K(k) \rightarrow 0 .
$$

Now $F$ is a sum of line bundles and so therefore is $\left(\wedge^{\prime} F\right)(k)$ for all $l$. In fact,

$$
\left(\wedge^{\prime} F\right)(k)=\bigoplus_{1 \leqslant j_{1}<\cdots<j_{1} \leqslant n} \mathcal{O}_{\mathbf{P}_{c}^{n_{1}} \cdot 1}\left(k-\left(d_{j_{1}}+\cdots+d_{j_{1}}\right)\right) .
$$

It is easy to show that $H^{0}(K(k)) \cong \bigoplus_{l=2}^{n} H^{0}\left(\left(\bigwedge^{\prime} F\right)(k)\right)$ and the result follows.

\section{BIBLIOGRAPHY}

1. Mei-Chu Chang, Stable rank 2 bundles on $\mathbf{P}^{3}$ with $c_{1}=0, c_{2}=4$, and $\alpha=1$, Math. Z. 184 (1983), 407-415.

2. C. K. Chui and R. H. Wang, Multivariate spline spaces, J. Math. Anal. Appl. (to appear).

3. A. Grothendieck, Sur la classification des fibrés holomorphes sur la sphère de Riemann, Amer. J. Math. 79 (1957), 121-138.

4. R. Hartshorne, On the classification of algebraic space curves, Vector Bundles and Differential Equations (Nice, 1979), Birkhäuser, Boston, Mass., 1980.

5. C. Okonek, M. Schneider and H. Spindler, Vector bundles on complex projective spaces, Birkhäuser, Boston, Mass., 1980.

6. S. Shatz, On subbundles of vector bundles over $\mathbf{P}^{1}$, J. Pure Appl. Algebra 10 (1977), 315-322.

7. P. Stiller, Certain reflexive sheaves on $\mathbf{P}_{\mathbf{C}}^{n}$ and a problem in approximation theory, Trans. Amer. Math. Soc. 279 (1983), 125-142.

8. B. L. Van der Waerden, Algebra, vol. 1, 7th ed., Ungar, New York, 1970.

9. C. Micchelli and W. Dahmen, On the local linear independence of translates of a box spline, Studia Math. (to appear).

10. Math. 10 (1984),

11. , On the optimal approximation rates for criss-cross finite element spaces, Comp. Applied , On the solutions of certain systems of partial difference equations and linear dependence of translates of box splines, IBM Research Report, IBM Thomas J. Watson Research Center, Yorktown Heights, N. Y.

12. E. M. Stein and G. Weiss, Introduction to Fourier analysis on Euclidean spaces, Princeton Univ. Press, Princeton, N. J., 1971.

13. R. Hartshorne, Algebraic geometry, Graduate Texts in Math., vol. 52, Springer-Verlag, Berlin and New York, 1977.

Department of Mathematics, Texas A \& M University, College Station, TeXas 77843 - 3368

Current address: Department of Mathematics, Louisiana State University, Baton Rouge, Louisiana 70803-4918 